BULL. AUSTRAL. MATH. SOC.

$08 B 10,(08 A 05)$

VOL. $32(1985), 33-44$.

\title{
INJECTIVITY AND RELATED CONCEPTS \\ IN MODULAR VARIETIES \\ I. TWO COMMUTATOR PROPERTIES
}

\author{
EMIL W. KISS
}

\begin{abstract}
The first property occurred in the investigation of directly representable varieties, and was named $C 2$ by $R$. McKenzie, the second one is new. Our analysis is independent of injectivity. However, in the forthcoming second part of this paper we are going to prove that varieties with enough injectives satisfy both properties, and shall use intensively the results proved here.
\end{abstract}

\section{Notation and quoted results}

The paper is intended to be self-contained modulo the results of this section. However, we refer the reader to Freese and McKenzie [1] and Gumm [4] for the background in commutator theory, and to Grätzer [3] for general terminology.

Throughout the paper we assume that all algebras considered are in a fixed modular variety $V$. The smallest and greatest congruence of an algebra $A$ is denoted by $0_{A}$ and ${ }_{A}$, respectively, indices are often omitted. The join and meet of the congruences $\theta$ and $\psi$ are denoted by $\theta+\psi$ and $\theta \psi$. The notation $(a, b) \in \theta, a \equiv b(\theta)$ and $a \theta b$ are equivalent. The smallest congruence of an algebra $A$ containing a given

Received 3 December 1984.

Copyright Clearance Centre, Inc. Serial-fee code: 0004-9727/85 $\$$ A2.00 + 0.00 . 
$H \subseteq A \times A$ is denoted by $\mathrm{Cg}_{A}(H)$, where $A$ is the underlying set of $A$; $\operatorname{Cg}(a, b)$ stands for $\mathrm{Cg}_{A}(\{(a, b)\})$. The restriction of an $\alpha \in$ Con $A$ to a $\mathrm{B} \leq \mathrm{A}$ is $\alpha / \mathrm{B}$. The subalgebra generated by the set $H$ is $(H)$. If $f: A \rightarrow B$ is a homomorphism, and $C \leq A, \gamma \in$ Con $C$, then the image of $C$ and $\gamma$ under $f$ is $f^{\gg c}$ and $f^{\gg} \gamma$, or simply $C^{\gg}$ and $\gamma^{\gg}$ if it is clear that the homomorphism we consider is $f$.

The smallest nontrivial congruence of a subdirectly irreducible algebra $S$ is called the monolith of $S$, and is denoted by $\mu(S)$. An algebra $A$ is called finitely subdirectly irreducible, if $\alpha \beta=0$ implies $\alpha=0$ or $B=0$ for any two congruences of $A$. We use the abbreviations Si and FSi for these concepts.

The join $V_{1} \vee V_{2}$ of two varieties is $V\left(V_{1} \cup V_{2}\right)$, where the operator $V$ stands for HSP. We use also the operators $P_{S}$ (subdirect products), $P_{f}$ (products of finitely many components), $P_{s f}$ (subdirect products of finitely many components), $\mathrm{p}^{2}$ (direct squares), $\mathrm{P}_{u}$ (ultraproducts), D (direct unions), $\mathrm{Si}(K)$ (Si elements of the class $K$ ). Logical conjunction is often denoted by + , for example, $C D+C P$ stands for arithmetical varieties.

LEMMA 1.1. (1) $S P(K) \subseteq P_{S} S(K)$.

(2) The finite elements of $\mathrm{SP}(K)$ are in $\mathrm{SP}_{f}(K)$.

(3) The free algebra $F_{V(K)}(X)$ of $V(K)$ generated by the set $X$ is in $S P(K)$.

(4) $S(K) \subseteq H_{S}(K)$ (see Grätzer [3], Theorem 23.3).

Let $A \leq A_{1} \times A_{2}, \quad \alpha_{i} \in \operatorname{Con} A_{i}(i=1,2)$. The product of the congruences $\alpha_{1}$ and $\alpha_{2}$ on $A$ is defined by

$$
\left(a_{1}, a_{2}\right)\left(\alpha_{1} \times \alpha_{2}\right)\left(b_{1}, b_{2}\right) \text { if and only if } a_{1} \alpha_{1} b_{1} \text { and } a_{2} \alpha_{2} b_{2} \text {. }
$$

Congruences of this form are called product congruences. If $A$ is a subdirect subalgebra of $A_{1} \times A_{2}$, then $\alpha \in$ Con $A$ is a product congruence 
if and only if $\alpha=\left(\alpha+\eta_{1}\right)\left(\alpha+\eta_{2}\right)$, where $\eta_{i}$ is the kernel of the $i$ th projection.

DEFINITION 1.2. An algebra $A$ is called affine if there exists an Abelian group structure $(A,+,-, 0)$ on its underlying set such that

(i) each operation $f$ of $A$ is affine, that is, $f-c_{f}$ is a group homomorphism with respect to + for a suitable constant $c_{f} \in A$,

(ii) $x-y+z$ is a term function of $\mathrm{A}$.

Affine algebras of a given type form a variety, and are polynomially equivalent to modules over associative rings.

DEFINITION 1.3 (Gumm [4]). Let $A$ be an algebra, $\alpha, \beta \in$ Con $A$. Define

$$
\begin{aligned}
\Delta_{\alpha, \beta} & =\mathrm{Cg}_{\alpha}(\{(a a, b b): a \beta b\}), \\
{[\alpha, \beta] } & =\left\{(a, b): a a \Delta_{\alpha, \beta} a b\right\} .
\end{aligned}
$$

Here $a a, b b$ and $a b$ abbreviate the corresponding ordered pairs, and $\alpha$ is considered as a subalgebra of $A \times A .[\alpha, \beta]$ is the commutator of $\alpha$ and $\beta$.

THEOREM 1.4 (see Gumm [4]). Let $V$ be a congruence modular variety, $A, B \in V, \alpha, \beta, \beta_{i} \in \operatorname{Con} A(i \in I)$. Then the following hold:

(1) $[\alpha, \beta]=[\beta, \alpha] \in \operatorname{Con} A$;

(2) $[\alpha, \beta] \leq \alpha \beta ;$

(3) $\left[\alpha, \Sigma \beta_{i}\right]=\Sigma\left[\alpha, \beta_{i}\right]$;

(4) for any epimorphism $f: A \rightarrow B$ we have $f^{\gg}[\alpha, \beta]=\left[f^{\gg} \alpha, f^{\gg}\right]$;

(5) $A$ is affine if and only if $\left[I_{A}, 1_{A}\right]=O_{A}$.

DEFINITION 1.5 (Hagemann and Herrmann [5]). An algebra $A$ is called neutral if $A$ satisfies the commutator identity $[x, y]=x y$ (for each pair of congruences). An $\alpha \in$ Con $A$ is perfect if $[\alpha, \alpha]=\alpha$. The algebra $A$ is prime if $[\alpha, \beta]=0$ implies $\alpha=0$ or $\beta=0$, and 
semiprime if $[\alpha, \alpha]=0$ implies $\alpha=0$. A congruence $\alpha$ is prime (semiprime) if and only if $A / \alpha$ is.

THEOREM 1.6 (Hagemann and Herrmann [5]). The following are equivalent for an algebra. $A \in V$ :

(1) A is neutral;

(2) each congruence of $A$ is perfect;

(3) if $\alpha \in \operatorname{Con} A$, then $\operatorname{Con} \alpha$ is distributive.

The class of all neutral algebras of $V$ is closed under $\mathrm{D}, \mathrm{H}$ and $\mathrm{P}_{\mathrm{sf}}$.

THEOREM 1.7 (Hagemann and Herrmann [5]). A congruence $\alpha$ of an algebra is semiprime if and only if it is the meet of prime congruences.

THEOREM 1.8 (Generalized Jonsson's Theorem, see Hagemann and Herrmann [5]). Let $A_{i}(i \in I)$ be algebras of $V$, B a subalgebra of their direct product and $\alpha$ a prime congruence of $B$. Then there is an ultrafizter $U$ on the index set $I$ such that the corresponding congruence $\mathrm{Cg}_{\mathrm{B}}(u) \leq \alpha$. Consequently, the prime algebras of $V(K)$ are contained in $\operatorname{HSP}_{u}(K)$ for any $K \subseteq V$.

\section{The two properties}

The first property, named $C 2$, seems to be the "join" of affineness and neutrality, the second one, called $S$, is a sort of commutator extension property saying that « forming the commutator 》 commutes with « restricting congruences to subalgebras 》.

PROPOSITION 2.1. The following are equivalent for an algebra A :

(1) $\mathrm{A} F[x, y]=x y[1,1]$;

(2) $\mathrm{A} F[x, x]=x[1,1]$;

(3) if $a[1,1] b$ then $\mathrm{Cg}(a, b)$ is perfect;

(4) $\mathrm{A} F[x, y z]=[x, y] z ;$

(5) $A \neq[x, y]=[x, 1] y$.

Proof. (1) $\Rightarrow(4),(1) \Leftrightarrow(5)$ and (1) $\Leftrightarrow(6)$ are evident. (4) $\Rightarrow$ (3) holds, since $x$ is a join of principal congruences. For $(3) \Rightarrow(2)$ apply 
(3) to $x[1,1]$, and for (2) $\Rightarrow$ (1) apply (2) to $x y$.

DEFINITION 2.2. An algebra $A$ has property $C 2$ if any of the conditions in Proposition 2.1 holds in $A$, and has property $S$ if for arbitrary $B \leq A$ and $\alpha, B \in \operatorname{Con} A$ we have $[\alpha / B, B / B]=[\alpha, \beta] / B$.

The property $S$ will always be investigated together with $C 2$. In this case it is enough to require only the special case $\alpha=\beta=1$.

PROPOSITION 2.3. If all the subalgebras of $A$ are $C 2$, and for every $B \leq A$ we have $\left[I_{B}, I_{B}\right]=\left[I_{A}, I_{A}\right] / B$, then $A$ satisfies $S$.

A class of algebras is said to satisfy $C 2 \quad(S)$ if so do all of its members. Neutral algebras are clearly $C 2$, and affine ones are $C 2+S$. Further examples are found in the second part of the present paper.

\section{Preservation}

We investigate the behaviour of our properties with respect to standard algebraic constructions.

PROPOSITION 3.1. The class of $C 2$ algebras is closed under the operators $H, D$ and $P_{\text {sf }}$.

Proof. Since the assertion is straightforward for $D$ and $H$, it suffices to verify the following lemma.

LEMMA 3.2. Suppose that $\mathrm{A}_{1}$ and $\mathrm{A}_{2}$ are $\mathrm{C}_{2}$ algebras, and $\mathrm{B}$ is a subdirect subalgebra of their direct product. Then

(1) B satisfies C2,

(2) if $\alpha \leq\left[I_{B}, l_{B}\right]$, then $\alpha$ is a product congruence, in particular $\left[I_{B}, I_{B}\right]=\left(\left[I_{A_{1}}, I_{A_{1}}\right] \times\left[I_{A_{2}}, I_{A_{2}}\right]\right) / B$.

Proof. To show (2), let $\eta_{1}$ and $n_{2}$ be the projection kernels on $B$ and consider the sublattice of Con B generated by $\alpha, \eta_{1}$ and $\eta_{2}$. It is a homomorphic image of the following lattice which is the free modular lattice generated by $\alpha, \eta_{1}$ and $\eta_{2}$ subject to the relation $\eta_{1} \eta_{2} \leq \alpha$ (see Grätzer [2]): 


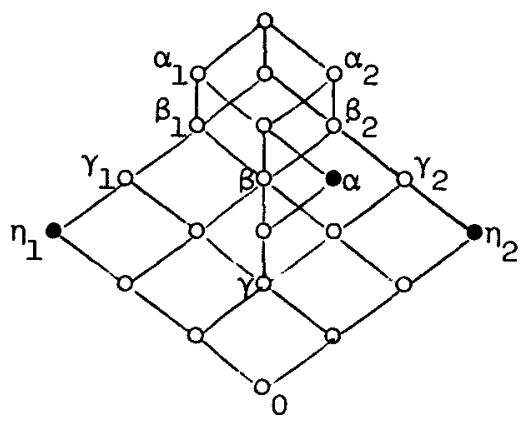

Let $\alpha_{i}=\alpha+\eta_{i} \quad(i=1,2)$, we have to prove that $\alpha=\alpha_{1} \alpha_{2}$. Consider the diamond between $\beta$ and $\gamma$. An easy computation shows $[\beta, B] \leq \gamma$. Now $B / \eta_{1}$ is isomorphic to $A_{1}$, so this factor is $C 2$. Hence $\alpha \leq\left[I_{B}, I_{B}\right]$ implies that there is no affine interval between $\alpha_{1}$ and $\eta_{1}$. Thus $\beta_{1}=\gamma_{1}$, consequently $\beta=\gamma$, which yields $\alpha=\alpha_{1} \alpha_{2}$. So (2) holds.

To get (I) observe that if $\alpha \leq\left[I_{B}, I_{B}\right]$, then $\alpha$ and $[\alpha, \alpha]$ are both product congruences by (2). As $A_{1}$ and $A_{2}$ have $C 2$, the image of $\alpha$ under the projections is perfect. Hence $\alpha$ and $[\alpha, \alpha]$ are the product of the same congruences, and therefore they are equal.

Unfortunately, in the previous proof we had to use the perfectness of $B_{1}^{\gg}$ and $B_{2}^{\gg}$. But if $B$ is the direct product of $A_{1}$ and $A_{2}$, then $\eta_{1}+\eta_{2}=1$, hence $\beta_{i}=\gamma_{i}$, so our reasoning yields

LEMMA 3.3. Let $\alpha \in \operatorname{Con}\left(A_{1} \times A_{2}\right), A_{1}, A_{2}$ arbitrary. If the image of $\alpha$ under the projections is perfect, then $\alpha$ is perfect as well.

PROPOSITION 3.4. The property $C 2+S$ is closed under $H, D, P_{S f}$. If all the subalgebras of $\mathrm{A}$ have $\mathrm{C} 2$, and $\mathrm{A}$ has $S$, then every $\mathrm{B} \leq \mathrm{A}$ has $S$.

Proof. The statement is straightforward for $D, H$, and the rest is an easy consequence of Proposition 2.3 and Lemma 3.2.

\section{Subdirectly irreducibie algebras}

We give characterisations of $C 2$ and $C 2+S$ varieties by means of 
conditions imposed on their subdirectly irreducible members. Sometimes these conditions involve $P_{u}(K)$ for some class $K$. Though such a condition is not very easy to handle and check in general, it becomes clear when $K$ is axiomatic, or, in particular, if $K$ is a finite set of finite algebras.

PROPOSITION 4.1. A finitely subdirectly irreducible c2 algebra is either prime or affine.

Proof. If $[1,1] \neq 0$, then $0=[\alpha, \beta]=\alpha \beta[1,1]$ yields $\alpha=0$ or $B=0$ by the FSi property.

PROPOSITION 4.2. A variety $V$ has $C 2$ if and only if each $S i$ nember of $V$ is affine or prime.

Proof. Suppose that $[1,1] \geq \alpha \in \operatorname{Con} A, A \in V$, and the Si members of $V$ are either affine or prime. If $[\alpha, \alpha]<\alpha$, then there is a congruence $\theta$ of $A$ such that $A / \theta$ is $\mathrm{Si}, \theta \geq[\alpha, \alpha]$, but $\theta \nsupseteq \alpha$. Then $\theta+\alpha \notin \alpha$, so $[\theta+\alpha, \theta+\alpha] \leq \theta+[\alpha, \alpha]=\theta$ shows that $A / \alpha$ is not prime. Hence it is affine, yielding $\theta \geq[1,1] \geq \alpha$, which is a contradiction. The converse is Proposition 4.1.

THEOREM 4.3. A variety $V$ satisfies $C 2+S$ if and only if

(i) $\mathrm{Si}(V)$ satisfies $C 2$ and

(ii) $P_{u} S i(V)$ satisfies $S$.

Proof. Suppose $V$ satisfies $(i)$ and $(i i)$. Then $V$ is $C 2$ by Propositions 4.1 and 4.2. The FSi algebras of $V$ are either affine, or contained in $\mathrm{HSP}_{\mathrm{U}} \mathrm{Si}(V)$ by the Generalized Jónsson's Theorem, so they have $S$ by Proposition 3.4. We are going to find a method of factorising algebras into FSi ones. Call a congruence $\delta$ subperfect if every $\delta^{\prime} \leq \delta$ is perfect.

LEMMA 4.4. If $\alpha, \beta, \delta \in \operatorname{Con} A$ and $\delta$ is subperfect, then $\delta+\alpha \beta=(\delta+\alpha)(\delta+\beta)$.

Proof. By setting $\delta^{\prime}=\delta(\alpha+\beta)$ we obtain

$$
\delta^{\prime}=\left[\delta^{\prime}, \delta^{\prime}\right] \leq[\delta, \alpha+\beta] \leq \delta \alpha+\delta \beta .
$$

Hence $\delta(\alpha+\beta)=\delta \alpha+\delta \beta$. It is an elementary result of modular lattice theory that this implies our assertion (see Grätzer [2]). 
LEMMA 4.5. Let $\delta$ be a subperfect congruence of $a \mathrm{~B} \leq \mathrm{A}$. If $\delta \nsupseteq \gamma / B$ for some $\gamma \in$ Con $A$, then $\delta^{\gg} \notin(\gamma / B) \gg$ holds in an appropriate FSi factor of $A$.

Proof. Choose $(a, b) \in(\gamma / B)-\delta$, and let $\theta_{0}$ be maximal among the congruences $\theta$ of $A$ satisfying $(a, b) \neq \theta / B+\delta$. Then $A / \theta_{0}$ is a FSi factor of $A$ by Lemma 4.4 , and the image of $(a, b)$ yields $\delta^{\gg} \nsupseteq(\gamma / B) \gg$.

Now the proof of Theorem 4.3 is finished by the following corollary, which comes from Lemma 4.5 with $\delta=\left[I_{B}, I_{B}\right]$ and $\gamma=\left[I_{A}, I_{A}\right]$.

COROLLARY 4.6. Let $\mathrm{B} \leq \mathrm{A}$ be $\mathrm{C2}$ algebras and assume that every prime factor of $A$ satisfies $S$. Then $[\alpha, B] / B=[\alpha / B, B / B]$ holds for all congruences $\alpha, \beta$ of $A$. In particular, $a$ C2 variety has $S$ if and only if its prime algebras have $S$.

\section{Generator classes}

Given a class $K$ of algebras we would like to know whether $V(K)$ satisfies $C 2$ or $C 2+S$. For the first question - even in the case of neutrality - we have only a partial answer, so we start with a problem.

PROBLEM 5.1. Let $K$ be a class of algebras such that $S P_{U}(K)$ is neutral (C2). Does it follow that $V(K)$ is neutral (C2) ?

As partial solutions we can state

PROPOSITION 5.2. Let $K$ be a class of algebras. If $\mathrm{P}_{u}(K)$ is neutral (C2), then so is $P(K)$.

PROPOSITION 5.3. Let $K$ be a class of algebras, $V=V(K)$. If $\mathrm{SP}_{u}(K)$ satisfies $C 2+S$, and $\mathrm{F}_{V}(2)$ is either finite or $C 2$, then $V$ has $C 2+S$.

PROPOSITION 5.4. Let $K$ be a finite set of finite algebras. Then $V(K)$ has $C 2(C 2+S)$ if and only if $S(K)$ does.

Proposition 5.4 is clear: $V=\mathrm{DHP}_{S f} \mathrm{~S}(K)$, hence Propositions 3.1 and 3.4 apply. For Proposition 5.2 we need a definition. 
DEFINITION 5.5. Let $A_{i}(i \in I)$ be algebras, $B \leq \pi\left\{A_{i}: i \in I\right\}$, and $a, b \in B$. A subset $X$ of $I$ is called a set of perfectness for $a, b$ and $B$, if projecting $B$ to $\Pi\left\{A_{i}: i \in X\right\}$, the image of $\mathrm{Cg}_{\mathbf{B}}(a, b)$ is perfect.

In what follows, the kernel of this projection is denoted by $\mathrm{Cg}_{B}(X)$. If $U$ is an ultrafilter on $I$, the corresponding congruence on $B$ is named $\mathrm{Cg}_{B}(U)$. Algebras of the form $B / \mathrm{Cg}_{B}(U)$ are called subultraproducts.

LEMMA 5.6. With the notation above, $I$ is the join of finitely many sets of perfectness for $a, b$ and $B$ if and only if the image of $\mathrm{Cg}_{\mathrm{B}}(a, b)$ is perfect in all the sub-ultraproducts formed from $\mathrm{B}$.

Proof. If $I$ is the join of a finite number of sets of perfectness, and $U$ is an ultrafilter on $I$, then one of these sets, say $X \in U$. Hence $\mathrm{Cg}(X) \leq \mathrm{Cg}(u)$, thus the image of $\operatorname{Cg}(a, b)$ in the sub-ultraproduct is an image of a perfect image of $\operatorname{Cg}(a, b)$. Conversely, let $F$ be the ideal of finite joins of sets of perfectness, and $u$ an ultrafilter disjoint from $F$. The perfectness of a principal congruence can be described by finitely many equations, as it turns out from the definition of the commutator. So if the image of $\operatorname{cg}(a, b)$ is perfect in the subultraproduct corresponding to $U$, then - since $U$ is closed under finite intersections - the image of $\mathrm{Cg}(a, b)$ is perfect in $\mathrm{B} / \mathrm{Cg}(X)$ for an appropriate $X \in U$. But then $X$ is a set of perfectness in $U$, and this is a contradiction.

Now Lemma 3.3 asserts that if $B$ is the whole direct product, then the join of finitely many sets of perfectness is a set of perfectness again. So the neutral case of Proposition 5.2 is clear by applying Lema 5.6 to all pairs $(a, b)$ of $B$, while for the case of $c 2$ one has to consider the pairs in $\left[1_{B}, 1_{B}\right]$.

Finally, we show Proposition 5.3. Note first that if $F_{V}(2)$ is finite, then it is in $P_{S f} S(K)$, hence satisfies $C 2$ by Proposition 3.4 . We copy the proof of Theorem 4.3. Let $A_{i} \in K$, and 
$\mathrm{C} \leq \mathrm{B} \leq \mathrm{A}=\Pi\left\{\mathrm{A}_{i}: i \in I\right\}$. Suppose $(a, b) \in\left[\mathrm{I}_{\mathrm{B}}, \mathrm{I}_{\mathrm{B}}\right] / \mathrm{C}-\left[\mathrm{I}_{\mathrm{C}}, \mathrm{I}_{\mathrm{C}}\right]$. Then for $D=(a, b)$ we have $(a, b) \in\left[I_{A}, 1_{A}\right] / D-\left[I_{D}, 1_{D}\right]$. But $A$ has $C 2$ by Proposition 5.2 , $D$ has $C 2$, as it is a homomorphic image of $F_{V}(2)$, and the prime algebras have $S$ by the assumption. So Corollary 4.6 gives a contradiction. Hence,

$$
\left[I_{B}, 1_{B}\right] / C=\left[1_{C}, 1_{C}\right] \text {. }
$$

Now apply (*) to $\mathrm{C}=\langle a, b\rangle$ for some $a\left[I_{\mathrm{B}}, I_{\mathrm{B}}\right] b$. As $\mathrm{C}$ has $C 2$, we get the perfectness of $\operatorname{Cg}_{B}(a, b)$. Thus $B$ has $C 2$. Hence (*) and Proposition 2.3 show that $B$ has $C 2+S$. So $V=H S P(K)$ has $C 2+S$ by Proposition 3.4 .

\section{Joins of varieties}

We examine the behaviour of the join of two subvarieties of the fixed large modular variety with respect to our properties.

PROPOSITION 6.1. Let $V_{1}$ and $V_{2}$ be varieties. Then $V_{1} \vee V_{2}$ satisfies $C 2(C 2+S)$ if and only if both $V_{1}$ and $v_{2}$ do also. If $V_{1} \vee V_{2}$ has $C 2$, then each of its elements has a subdirect decomposition into three factors: one from $V_{1}$, one from $V_{2}$, and the third factor is affine.

Proof. Since $V_{1} \vee V_{2}=H_{s f}\left(V_{1} \cup V_{2}\right)$, the first statement follows from Propositions 3.1 and 3.4. The Generalized Jónsson's Theorem (applied with $a^{*}$ 2-element index set) shows that the prime algebras of $V_{1} \vee V_{2}$ are in $V_{1} \cup V_{2}$. So the second assertion follows from Proposition 4.1 and the Birkhoff theorem.

\section{Disconnected varieties}

We look for conditions under which a variety is the join of an affine and a congruence distributive variety. These varieties have been investigated by Herrmann [6], their structure is described in Freese and McKenzie [1]. 
DEFINITION 7.1. An algebra is called disconnected, if it is the direct product of a neutral and an affine algebra. A variety is disconnected if and only if it is the join of an affine and a congruence distributive variety .

THEOREM 7.2. Let $K$ be the class of all neutral Si algebras of $a$ variety $V$. The following are equivalent for $V$ :

(I) $V$ is disconnected;

(2) each algebra in $V$ is disconnected;

(3) the Si elements of $V$ are either affine or neutral, and $\mathrm{SP}_{\mathrm{u}}(\mathrm{K})$ is neutral.

Disconnected varieties satisfy $C 2+S$. If $F_{V}(3)$ is finite, then (3) can be replaced by

(3') the Si elements of $V$ are either affine, or each of their subalgebras is neutral.

REMARK. In the second part of the present paper we give an example of a locally finite $C 2+S$ variety, which is not disconnected.

Proof. (1) $\Rightarrow(2)$. This is Hermann's result (see [6]).

$(2) \Rightarrow(3)$. A disconnected Si algebra is either affine or neutral. Subdirect products of neutral algebras are semiprime by Theorem 1.7 , so they are neutral by (2). Now Lemma 1.1 (4) gives $\mathrm{SP}_{u}(K) \subseteq \mathrm{HP}_{\mathrm{S}}(K)$, so $S P_{u}(K)$ is neutral.

$(3) \Rightarrow(V$ satisfies $C 2+S) . V$ has $C 2$ by Proposition 4.2 . If an element of $P_{u} S i(V)$ is not affine, then it is in $P_{u}(K)$. Hence the nonaffine elements of $S P_{u} S i(V)$ are in $S P_{u}(K)$, so they satisfy $S$ by (3). Thus Theorem 4.3 applies.

$(3) \Rightarrow(1)$. Proposition 5.3 shows that $V_{1}=V(K)$ is neutral. Let $V_{2}$ be the subvariety of all affine algebras. Then $\operatorname{si}(V) \subseteq V_{1} \cup V_{2}$ by (3), so $v=v_{1} \vee v_{2}$.

$\left(3^{\prime}\right) \Rightarrow(3)$ provided $F_{V}(3)$ is finite. Let $V_{1}=V(K)$. The 
3-generated free algebra-over $V_{1}$ is finite, so it is in $P_{S f} S(K)$ by Lemma 1.1, which is congruence distributive by ( $\left.3^{\prime}\right)$ and Theorem 1.6. Thus $V_{1}$ is CD by Jónsson [7].

\section{References}

[1] R. Freese and R. McKenzie, "The commutator, an overview", preprint.

[2] G. Grätzer, General lattice theory (Birkhäuser Verlag, Basel, 1978).

[3] G. Grätzer, Universal algebra, second edition (Springer-Verlag, Berlin, Heidelberg, New York, 1979).

[4] H.-P. Gumm, "An easy way to the commutator in modular varieties", Arch. Math. (Basel) 34 (1980), 220-228.

[5] J. Hagemann and C. Herrmann, "A concrete ideal multiplication for algebraic systems and its relation to congruence distributivity", Arch. Math. (Basel) 32 (1979), 234-245.

[6] C. Herrmann, "Affine algebras in congruence modular varieties", Acta Sci. Math. (Szeged) 41 (1979), 119-125.

[7] B. Jónsson, "Algebras whose congruence lattices are distributive", Math. Scand. 21 (1967), 110-121.

Mathematical Institute of the Hungarian Academy of Sciences, 1364 Budapest, POB 127, Hungary. 